\title{
Phonocardiogram Signal Denoising Based on Non-negative Matrix Factorization and Adaptive Contour Representation Computation
}

\author{
Duong-Hung Pham, Sylvain Meignen, Nafissa Dia, Julie Fontecave-Jallon and Bertrand Rivet
}

\begin{abstract}
This letter introduces a new technique for phonocardiogram (PCG) signal denoising based non-negative matrix factorization (NMF) of its spectrogram and adaptive contour representation computation (ACRC) of its short-time Fourier transform (STFT). More precisely, NMFs on PCG and synchronous electrocardiogram (ECG) spectrograms are first used to filter out high-energy noises from PCG. Then, ACRC is performed on a low-pass filtered version of the STFT of the resulting signal to identify relevant time-frequency (TF) components which are subsequently used for signal retrieval. Numerical experiments conducted on a real database of noisy PCG signals (SiSEC2016) illustrate the superiority of the proposed method over state-ofthe-art techniques.
\end{abstract}

Index Terms-Time-frequency analysis, multimodality, ECG, PCG, NMF

\section{INTRODUCTION}

$\mathbf{C}$ ARDIOVASCULAR diseases (CVDs) are one of the world largest public health problems, causing the death of nearly 18 million people annually, accounting for one-third of deaths worldwide [1]. Many efforts have therefore been put on CVDs early diagnosis, mostly relying on techniques built on cardiac auscultation. These are non-invasive and lowcost, being based on the listening of the heart sounds using an acoustic stethoscope. Among many sounds produced by the heart during a cardiac cycle, the two following ones are the most audible. S1 is the sound created by the closing of atrioventricular valves corresponding to the beginning of ventricular systole, while S2 is associated with the closing of the semilunar valves during ventricular diastole [2], [3]. With the development of electronic stethoscopes, the graphical recording of heart sounds, called phonocardiogram (PCG) signals, can also be displayed on a digital computer, and then analyzed, to provide more insightful information on the condition of the heart [4]. Unfortunately, such signals are often severely contaminated by many different types of noises including subject movement or speech, ambient sources, stethoscope movement or lung sounds, making its analysis

This work has been partially supported by the LabEx PERSYVAL-Lab (ANR-11-LABX-0025-01) funded by the French program Investissements d'avenir.

D.-H. Pham and S. Meignen are with the Jean Kuntzmann Laboratory, Université Grenoble Alpes, 38401 Saint-Martin-d'Hères, France.

N. Dia and J. Fontecave-Jallon are with the TIMC-IMAG Laboratory, Université Grenoble Alpes, 38706 La Tronche, France.

B. Rivet is with the GIPSA-Lab Laboratory, UMR 5216, 38401 SaintMartin-d'Hères, France. quite difficult [5]. Various methods have been proposed in the literature to denoise PCG, among which those based on wavelet or STFT thresholding [6]-[9], or empirical mode decomposition (EMD) [10]-[13] are the most popular. However, all of them rely on the analysis of the PCG only but, to improve the denoising performance, it may be of interest to use extra non-invasive recordings, as for instance the electrocardiogram (ECG).

This idea was indeed investigated in [14], where the analysis of simultaneous PCG and ECG was carried out by first decomposing their respective spectrograms using non-negative matrix factorization (NMF) to obtain so-called activation functions associated with PCG and ECG. The analysis of their correlation lead to a PCG signal denoising algorithm, called NMFdenoising. Unfortunately, the procedure relied on a threshold, fixed $a$ priori, thus limiting its adaptivity. In another direction, a novel TF method, called adaptive contour representation computation (ACRC), was proposed to adaptively retrieve and denoise the modes of multicomponent signals (MCSs) [15][17]. While this technique cannot efficiently deal with highly energetic noises which are common in PCG signals, our goal is here to show that to apply it to a low-pass version of the signal obtained after application of NMF-denoising results in improved denoising performance and adaptivity.

We, therefore, describe our new technique for PCG denoising, which we coin NMF-ACRC, in Section II. Then, in Section III, we provide numerical simulations, carried out on the SiSEC2016 database [18], to demonstrate the improvement brought by the proposed method over existing ones.

\section{Proposed Method for PCG Denoising}

This section describes the proposed algorithm for PCG denoising called NMF-ACRC. It relies on the successive combination of a denoising procedure based on NMF, followed by ACRC. The principle of the denoising technique using NMF is to determine a Wiener filter to apply to the STFT of the original signal to get a first denoised signal. The denoising process is then improved by low-pass filtering (LPF) the former and then applying ACRC. The explicit block diagram of the proposed algorithm is depicted in Fig. 1, its two main steps, i.e. NMF and ACRC, being discussed in details hereafter. 


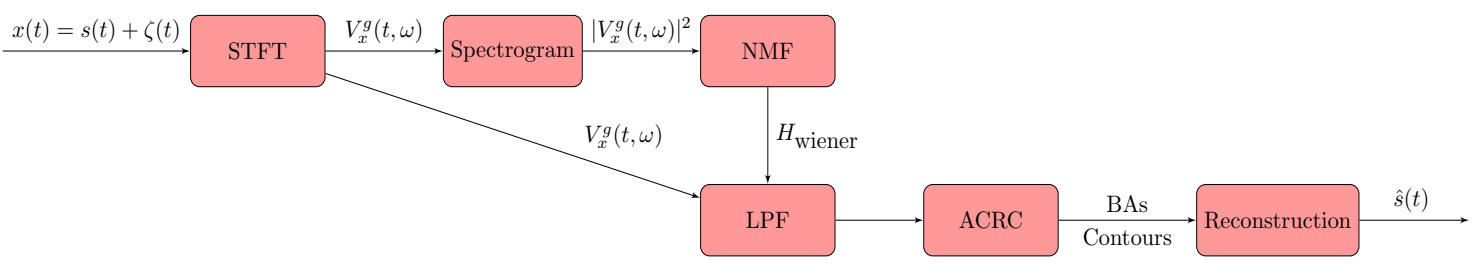

Fig. 1. Block diagram of NMF-ACRC algorithm.

\section{A. NMF-denoising: Filtering out High-energy Noises}

The key idea of denoising techniques based on NMF lies in that information of a single natural phenomenon can be acquired using different devices, called modalities [19], [20]. For instance, ECG corresponds to the recording of the electrical activity of the heart, while PCG to heart sounds. Modalities have similarities with one another, among which the most relevant is definitely quasi-periodicity, i.e. activation and inactivation periods are almost the same. NMFs of the spectrogram of simultaneous PCG and ECG enable to reflect this property by putting forward similar parameters, called shared factors [21]. In a nutshell, NMF approximates a $m$ by $n$ matrix $\mathbf{V}$ with non-negative elements by the product of two non-negative matrices $\mathbf{W}$ and $\mathbf{H}$ both with non-negative elements and with respective sizes $m \times k$ and $k \times n: \mathbf{V} \approx \mathbf{W H}$, where $k$, much smaller than $m$ or $n$, is called the number of estimated components. In that context, $\mathbf{H}$ is the shared factor which varies little across all the recordings of multimodal datasets.

NMFs applied to the spectrograms of physiological signals are of particular interest for identifying signals exhibiting similar temporal behaviors [22], since their shared factors are highly correlated. With this in mind, a procedure enabling the elimination of high-energy noises from noisy PCGs using synchronous ECGs was proposed in [14]. It consists of the following steps. First, one computes the spectrograms of noisy PCG and synchronous ECG, respectively denoted $\mathbf{V}_{x}$ and $\mathbf{V}_{\text {ecg }}$ (in our simulation, we consider the Gaussian window $\left.g(t)=\sigma^{-1} e^{-\pi \frac{t^{2}}{\sigma^{2}}}\right)$. Then, NMF is applied to $\mathbf{V}_{x}$, assuming

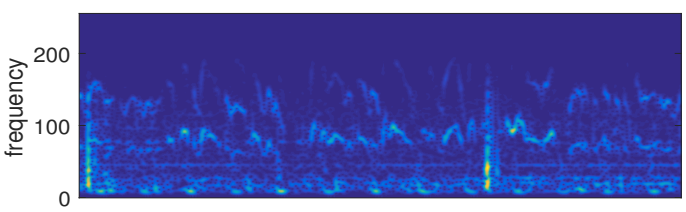

(a)

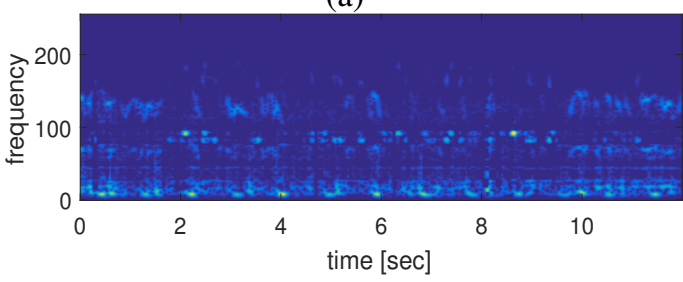

(b)

Fig. 2. Application of NMF-based algorithm on a noisy PCG: (a) noisy spectrogram $x$; (b) denoised spectrogram $\hat{s}_{1}$. $k=12$ (chosen number) components, i.e. $\mathbf{V}_{x}^{m, n}(t, \omega) \approx$ $\mathbf{W}_{x}^{m, 12}(\omega) \mathbf{H}_{x}^{12, n}(t)$, and to $\mathbf{V}_{e c g}$, assuming one component, i.e. $\mathbf{V}_{e c g}^{m, n}(t, \omega) \approx \mathbf{W}_{e c g}^{m, 1}(\omega) \mathbf{H}_{e c g}^{1, n}(t)$ (the sizes of the matrices are added as superscript to emphasize those of $\mathbf{H}_{x}$ and $\mathbf{H}_{e c g}$ are different). One then computes the cross-correlation between $\mathbf{H}_{x}^{k, \cdot}(t)$ and $\mathbf{H}_{e c g}^{1, \cdot}(t)$, for each $k$ : when it is larger than some chosen threshold $\lambda$, the corresponding $\mathbf{H}_{x}^{k, \cdot}(t)$ is associated with PCG (we define this way $\mathbf{H}_{\hat{s}_{1}}^{12, n}(t)$ ), and noise otherwise (corresponding to the definition of $\mathbf{H}_{\hat{\zeta}_{1}}^{12, n}(t)$, such that $\left.\mathbf{H}_{\hat{s}_{1}}^{12, n}(t)+\mathbf{H}_{\hat{\zeta}_{1}}^{12, n}(t)=\mathbf{H}_{x}^{12, n}(t)\right)$. This finally enables an estimation of the spectrograms of the PCG and of the noise through: $\mathbf{V}_{\hat{s}_{1}}(t, \omega)=\mathbf{W}_{x}^{m, 12}(\omega) \mathbf{H}_{\hat{s}_{1}}^{12, n}(t)$ and $\mathbf{V}_{\hat{\zeta}_{1}}(t, \omega)=\mathbf{W}_{x}^{m, 12}(\omega) \mathbf{H}_{\hat{\zeta}_{1}}^{12, n}(t)$.

To obtain a denoised version of the original signal, from the estimated spectrograms one builds a Wiener filter as follows:

$$
H_{\text {wiener }}=\frac{\mathbf{V}_{\hat{s}_{1}}(t, \omega)}{\mathbf{V}_{\hat{s}_{1}}(t, \omega)+\mathbf{V}_{\hat{\zeta}_{1}}(t, \omega)},
$$

which, applied to the STFT of the original signal, leads to a denoised PCG signal, which is denoted $\hat{s}_{1}$ in the sequel.

To illustrate the principle of the algorithm just described, we first depict, in Fig. 2 (a), the spectrogram of a noisy PCG in which two high-energy noise pulses show up at times 0.2 second and 8.1 seconds, along with the denoised spectrogram using the just mentioned technique, in Fig. 2 (b). We notice that the latter removes the two high-energy noise pulses from the original PCG.

However, this result can still be improved by selecting the most relevant coefficients in the denoised spectrogram: this can be done by using the so-called ACRC as a post-processing step. A brief summary of such a technique and how to apply it in the studied context is the subject of the following section.

\section{B. ACRC-denoising: Components Estimation and Signal Re- trieval}

The ACRC introduced in [16], [17] is computed on a lowpass filtered (LPF) version of $\hat{s}_{1}$ with a cutoff frequency $80 \mathrm{~Hz}$ to enable not only the elimination of high-frequency noises but also a significant reduction of the computational cost of ACRC. From now on, we denote $\hat{s}_{1, L P F}$ such a signal. In what follows, the STFT of signal $x$ using the same Gaussian window $g$ as previously is denoted by $V_{x}^{g}$, and the principle of ACRC applied $\hat{s}_{1, L P F}$ is detailed hereafter. 


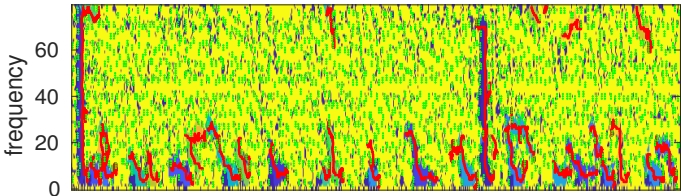

(a)

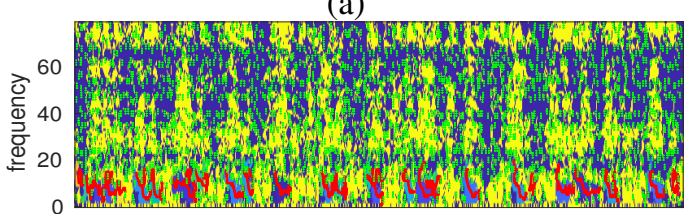

(b)

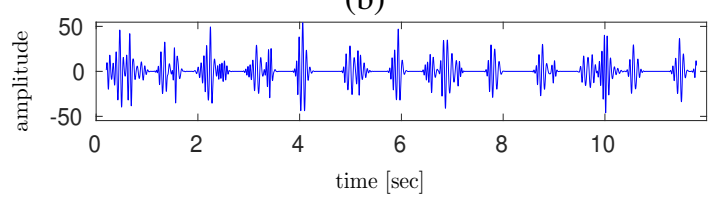

(c)

Fig. 3. Application of ACRC: (a) on the STFT of the initial noisy PCG displayed in Fig. 2 (a); (b): on $\hat{s}_{1, L P F}$; (c) reconstructed signal.

ACRC technique is based on the projection, in some specific direction, of the reassignment vector (RV) defined by [23].

$$
R V(t, \omega)=\left(\hat{\tau}_{\hat{s}_{1, L P F}}(t, \omega)-t, \hat{\omega}_{\hat{s}_{1, L P F}}(t, \omega)-\omega\right),
$$

where $\hat{\tau}_{\hat{s}_{1, L P F}}$ and $\hat{\omega}_{\hat{s}_{1, L P F}}$ are reassignment operators corresponding to [24]:

$$
\begin{aligned}
& \hat{\tau}_{\hat{s}_{1, L P F}}(t, \omega)=t+\Re\left\{\frac{V_{\hat{s}_{1, L P F}}^{t g}(t, \omega)}{V_{\hat{s}_{1, L P F}}^{g}(t, \omega)}\right\} \\
& \hat{\omega}_{\hat{s}_{1, L P F}}(t, \omega)=\omega-\frac{1}{2 \pi} \Im\left\{\frac{V_{\hat{s}_{1, L P F}}^{g^{\prime}}(t, \omega)}{V_{\hat{s}_{1, L P F}}^{g}(t, \omega)}\right\},
\end{aligned}
$$

$V_{\hat{s}_{1, L P F}}^{t g}, V_{\hat{s}_{1, L P F}}^{g^{\prime}}$ being respectively STFTs of $\hat{s}_{1, L P F}$ computed with windows $t \mapsto t g(t)$ and $g^{\prime}(t)$, and $\Re\{X\}$ and $\Im\{X\}$ are respectively the real and imaginary parts of complex number $X$.

When crossing a ridge associated with the TF signature of a component, RV undergoes a strong variation in its orientation. To locate these sudden changes, a strategy developed in [15], [23] consisted of two steps: the projection of RV in the direction associated with an angle $\theta$, and then contours points (CPs) were defined as the location of the sign changes of the projection. CPs are then linked to form so-called contours. To avoid both the issue of choosing $\theta$ and numerical instabilities, an adaptive technique, proposed in [17], defined CPs as the zeros of the projection of RV in a direction corresponding to the average of the orientation of RV over a squared neighborhood centered at the point of study. This resulted in a robust estimation of TF signatures for a wide class of MCSs. Therefore, we stick to that definition of contours in the present paper. Once the contours are computed, the BA associated with one contour corresponds to the set of points $(t, \omega)$ such that, at this location, RV points to that contour. Finally, signal reconstruction is performed by inverting the STFT considering only the coefficients belonging to these BAs.
We notice that the average number of contours detected is 3.5 contours/second: the quasi-periodicity of PCG can be reflected by ACRC and the total number of contours is close to $3.5 \mathrm{~N} / 1000, N$ being the signal length. We therefore display, in Fig. 3 (a) and (b), BAs along with the first 3.5N/1000 contours (in red) computed with ACRC on the non-filtered and filtered STFTs associated with the signals whose spectrograms are displayed in Fig. 2 (a) and (b), respectively. From Fig. 3 (a), it is clear that ACRC computes contours associated with the two high-energy impulse noises mentioned above, while after noise removal using NMF-based algorithm, the computed contours are apparently more relevant. Finally, we illustrate in Fig. 3 (c) the reconstruction of the PCG signal by selecting STFT coefficients associated with BAs that are displayed in Fig. 3 (b).

\section{NUMERICAL EXPERIMENTS AND DISCUSSION}

This section investigates the effectiveness of the proposed NMF-ACRC algorithm by comparing it with EMD [10], shifted-symmetrized-regularized hard-thresholding (SSR-HT) [9], NMF, and ACRC, on the SiSEC2016 database [18]. Note that it was shown in [9] that SST-HT outperforms the conventional hard-thresholding (HT) [25] and block-thresholding (BT) [26] for PCG denoising. Before going into the details of such comparisons, we first present the database and the evaluation criteria in the following sections. The Matlab code implementing the method and the scripts generating all figures are available at github.com/phamduonghung/NMF_ACRC2018.

\section{A. SiSEC2016 Database}

The numerical simulations are conducted on a real database that was already used during the sixth community-based Signal Separation Evaluation Campaign (SiSEC2016) [18]. PCGs were recorded with a cardiac microphone MLT210 on three healthy volunteers, while ECGs were simultaneously acquired by PowerLab instrument. Such signals sampled at $1 \mathrm{KHz}$ were then passed through a 15 to $300 \mathrm{~Hz}$ band-pass filter. In total, sixteen such synchronous PCGs and ECGs were acquired, lasting from ten seconds to more than a minute. In a second time, PCGs were artificially contaminated by different real interference (radio, cough, pseudo-periodic breathing noise, etc.).

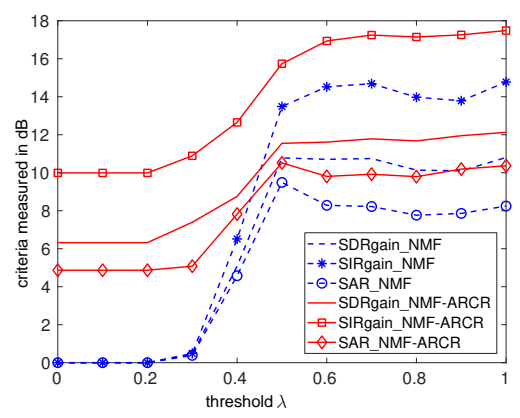

Fig. 4. SDR, SIR gains and SAR (measured in dB) for different crosscorrelation threshold values $\lambda$ when applying either NMF or NMF-ACRC to the noisy PCG signal displayed in Fig. 2 (a). 


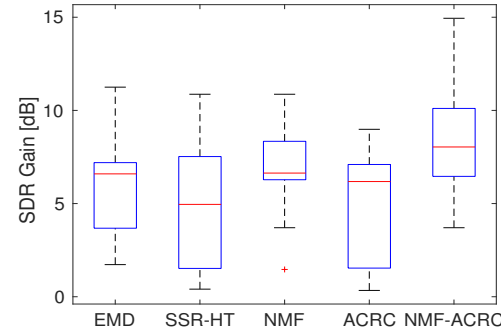

(a)

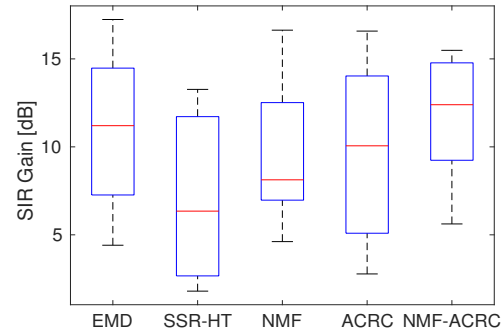

(b)

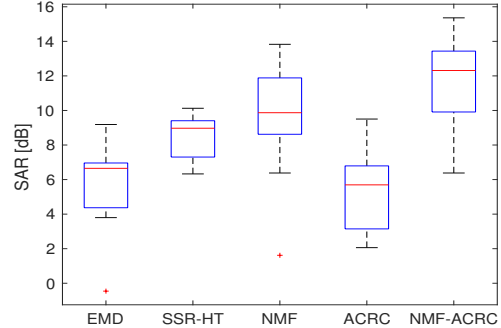

(c)

Fig. 5. Denoising performance (measured in $\mathrm{dB}$ ) of the tested techniques on the database SiSEC2016 (threshold $\lambda=0.75$ chosen optimally in [14]): (a) SDR gain; (b) SIR gain; (c) SAR.

\section{B. Evaluation Criteria}

In what follows, a quantitative measurement based on BSS Eval Toolbox [27] is used for the assessment of the denoising performance of the different methods. It consists in decomposing each estimated signal into a number of contributions associated with the target signal, interference of the unwanted sources and artifacts. This corresponds to the three following evaluation criteria expressed in decibels $(\mathrm{dB})$.

- Signal-to-Distortion Ratio (SDR): measures globally the level of all error terms.

- Signal-to-Interference Ratio (SIR): estimates the level of interference from all the other interfering sources.

- Signal-to-Artifacts Ratio (SAR): estimates the level of algorithmic artifacts and the linearity separation of the algorithm.

It should be noted that when using SDR and SIR, the gain between each of them obtained on the denoised (output) and initial noisy (input) signals is computed. For instance, SDR gain = output SDR-input SDR.

\section{Sensitivity to Cross-Correlation Threshold of NMF-ACRC}

As highlighted in Section II-A, the main drawback of NMF based denoising is that the threshold $\lambda$ needs to be manually set to separate the noise and signal components. To illustrate how NMF-ACRC manages to alleviate this dependence on $\lambda$, we display, in Fig. 4, the three evaluation measures (SDR, SIR gains and SAR) obtained when using either NMF-based or NMF-ACRC algorithms, for different $\lambda$ and for the signal depicted in Fig. 2 (a). It is clear to see that all these measures obtained with the former are null when $\lambda$ is small, which means that PCG is not denoised at all in that case. Furthermore, they increase and then stagnate when some particular value for $\lambda$ is reached. In contrast, the evaluation measures obtained when using the latter are bigger and also more stable with respect to parameter $\lambda$, especially when $\lambda$ is small. This tells us that to use NMF-ACRC rather NMF enables to limitate the dependance on $\lambda$, which makes the proposed new method more adaptive.

\section{Comparison of Denoising Performance}

This section presents some comparisons of NMF-ACRC with some state-of-the-art techniques including EMD, SSR-
HT, NMF-based, and ACRC on the SiSEC2016 database. To this end, we display the distribution of the different evaluation criteria using the boxplot representation, where the central line (in red) indicates the median, the bottom and top edges of the box indicating the 25 th and 75 th percentiles, respectively [28]. Furthermore, the threshold $\lambda$ is set to 0.75 in NMFbased and NMF-ACRC algorithms, which is also the optimal value already determined in [14]. Bear in mind that $\lambda$ is the threshold put on the cross-correlation between $H_{x}^{k, n}$ and $H_{e c g}^{1, n}$ to tell whether the $k$ th component is related to noise or signal.

In Fig. 5, we depict the denoising results associated with each of the tested techniques in terms of SDR, SIR gains and SAR. At first glance, we remark that NMF-ACRC produces better results than the other studied methods for all the three evaluation criteria. More precisely, in Fig. 5 (a), SDR median gain obtained by using NMF-ACRC is $8.0 \mathrm{~dB}$, while it equals 6.6, 4.9, 6.6 and $6.2 \mathrm{~dB}$ with EMD, SSR-HT, NMF-based, and ACRC, respectively. Moving to Fig. 5 (b), the former leads to an SIR median gain of $12.4 \mathrm{~dB}$ bigger than those associated with the latter $(11.2,6.3,8.1$ and $10.0 \mathrm{~dB}$ for EMD, SSR-HT, NMF-based, and ACRC respectively). Finally, Fig. 5 (c) shows that SAR median with the proposed technique is $12.3 \mathrm{~dB}$, while the ones associated with the others are much smaller: $6.6,8.9,8.7$, and $5.7 \mathrm{~dB}$, respectively. All in all, for PCG denoising, these results plead in favor of mixing NMF with ACRC as is done in NMF-ACRC.

\section{CONCLUSION}

In this letter, we have introduced a new technique for PCG denoising based on the use of two different techniques NMF and ACRC. By mixing them, we managed to circumvent their intrinsic limitations resulting in a significant improvement in terms of denoising performance. In a nutshell, NMF was first applied to remove the high-energy noises from the initial noisy PCG signal, while ACRC was carried out as a post-processing step to estimate the TF signatures of the relevant components. These were subsequently used for signal reconstruction. Numerical experiments demonstrated the effectiveness of the proposed technique on a database of real PCG signals. Future work should now be devoted to answer the question whether the improvement in terms of PCG signal denoising brought by NMF-ARCR can be profitably used to improve early diagnosis of heart diseases. 


\section{REFERENCES}

[1] W. H. Organization, "Global status report on noncommunicable diseases 2017," World Health Organization, Tech. Rep., 2017.

[2] H. Koymen, B.-K. Altay, and Y. Z. Ider, "A study of prosthetic heart valve sounds," IEEE transactions on biomedical engineering, no. 11, pp. 853-863, 1987.

[3] J. R. Mitchell and J.-J. Wang, "Expanding application of the wiggers diagram to teach cardiovascular physiology," Advances in physiology education, vol. 38, no. 2, pp. 170-175, 2014.

[4] J. Singh and R.-S. Anand, "Computer aided analysis of phonocardiogram," Journal of Medical Engineering \& Technology, vol. 31, no. 5, pp. 319-323, jan 2007.

[5] D. Gradolewski and G. Redlarski, "Wavelet-based denoising method for real phonocardiography signal recorded by mobile devices in noisy environment," Computers in Biology and Medicine, vol. 52, pp. 119129, Sep 2014.

[6] S.-R. Messer, J. Agzarian, and D. Abbott, "Optimal wavelet denoising for phonocardiograms," Microelectronics Journal, vol. 32, no. 12, pp. 931-941, Dec 2001.

[7] P. Varady, "Wavelet-based adaptive denoising of phonocardiographic records," in Conference Proceedings of the 23rd Annual International Conference of the IEEE Engineering in Medicine and Biology Society. IEEE, 2001.

[8] A.-K. Kumar and G. Saha, "A discrete wavelet packet transform based approach for selection of artifact-free phonocardiogram segment," in Emerging Applications of Information Technology (EAIT), 2014 Fourth International Conference of. IEEE, 2014, pp. 83-88.

[9] D.-H. Pham and S. Meignen, "A novel thresholding technique for the denoising of multicomponent signals," in IEEE ICASSP, Calgary, Alberta, Canada, 2018.

[10] G. Rilling, P. Flandrin, P. Goncalves et al., "On empirical mode decomposition and its algorithms," in IEEE-EURASIP workshop on nonlinear signal and image processing, vol. 3. NSIP-03, Grado (I), 2003, pp. 8-11.

[11] O. Beya, B. Jalil, E. Fauvet, and O. Laligant, "Empirical modal decomposition applied to cardiac signals analysis," in Wavelet Applications in Industrial Processing VII, F. Truchetet and O. Laligant, Eds. SPIE, feb 2010.

[12] A. Gavrovska, M. Slavkovic, I. Reljin, and B. Reljin, "Application of wavelet and emd-based denoising to phonocardiograms," in Signals, Circuits and Systems (ISSCS), 2013 International Symposium on. IEEE, 2013, pp. 1-4.

[13] O. Beya, E. Fauvet, and O. Laligant, "Eda, approche non linéaire de débruitage des signaux cardiaques," in CORESA, 2013.

[14] N. Dia, J. Fontecave-Jallon, P.-Y. Gumry, and B. Rivet, "Application de la factorisation non-ngative des matrices (nmf) pour le dbruitage des signaux phonocardiographiques," XXVIeme Colloque GRETSI à JuanLes-Pins, Sep 2017.

[15] S. Meignen, T. Gardner, and T. Oberlin, "Time-frequency ridge analysis based on reassignment vector," in Proceedings of the 23st European Signal Processing Conference (EUSIPCO-15). EURASIP, 2015.

[16] S. Meignen, T. Oberlin, P. Depalle, P. Flandrin, and S. McLaughlin, "Adaptive multimode signal reconstruction from time-frequency representations," Phil. Trans. R. Soc. A, vol. 374, no. 2065, p. 20150205, 2016.

[17] D.-H. Pham and S. Meignen, "An adaptive computation of contour representations for mode decomposition," IEEE Signal Processing Letters, vol. 24, no. 11, pp. 1596-1600, Nov 2017.

[18] A. Liutkus, F.-R. Stter, Z. Rafii, D. Kitamura, B. Rivet, N. Ito, Nobutaka, and O. Fontecave, "The 2016 signal separation evaluation campaign," LVA/ICA, pp. 323 - 332, 2017.

[19] D. Lahat, T. Adali, and C. Jutten, "Multimodal data fusion: An overview of methods, challenges, and prospects," Proceedings of the IEEE, vol. 103, no. 9, pp. 1449-1477, sep 2015.

[20] F. Sedighin, M. Babaie-Zadeh, B. Rivet, and C. Jutten, "Multimodal soft nonnegative matrix co-factorization for convolutive source separation," IEEE Transactions on Signal Processing, vol. 65, no. 12, pp. 3179-3190, jun 2017.

[21] B. Rivet, M. Duda, A. Guérin-Dugué, C. Jutten, and P. Comon, "Multimodal approach to estimate the ocular movements during eeg recordings: a coupled tensor factorization method," in Engineering in Medicine and Biology Society (EMBC), 2015 37th Annual International Conference of the IEEE. IEEE, 2015, pp. 6983-6986.

[22] M. Niegowski and M. Zivanovic, "Ecg-emg separation by using enhanced non-negative matrix factorization," in Engineering in Medicine and Biology Society (EMBC), 2014 36th Annual International Conference of the IEEE. IEEE, 2014, pp. 4212-4215.

[23] Y. Lim, B. Shinn-Cunningham, and T. Gardner, "Sparse contour representations of sound," IEEE Signal Processing Letters, vol. 19, no. 10, pp. 684-687, Oct 2012

[24] F. Auger and P. Flandrin, "Improving the readability of time-frequency and time-scale representations by the reassignment method," IEEE Transactions on Signal Processing, vol. 43, no. 5, pp. 1068-1089, 1995.

[25] D. Donoho and I. Johnstone, "Ideal spatial adaptation via wavelet shrinkage," Biometrika, vol. 81, pp. 425-455, 1994.

[26] G. Yu, S. Mallat, and E. Bacry, "Audio denoising by time-frequency block thresholding," IEEE Transactions on Signal Processing, vol. 56, no. 5, pp. 1830-1839, May 2008.

[27] E. Vincent, R. Gribonval, and C. Févotte, "Performance measurement in blind audio source separation," IEEE transactions on audio, speech, and language processing, vol. 14, no. 4, pp. 1462-1469, 2006.

[28] R. McGill, J. W. Tukey, and W. A. Larsen, "Variations of box plots," The American Statistician, vol. 32, no. 1, pp. 12-16, 1978. 